\title{
BFFT quantization and dynamical solutions of a fluid field theory
}

\author{
C. P. Natividade* \\ Instituto de Física, Universidade Federal Fluminense, Avenida Litorânea s/n, Boa Viagem, Niterói, 24210-340 Rio de Janeiro, Brazil \\ and Departamento de Física e Química, Universidade Estadual Paulista, Avenida Ariberto Pereira da Cunha 333, Guaratinguetá, \\ 12500-000 São Paulo, Brazil \\ H. Boschi-Filho ${ }^{\dagger}$ \\ Instituto de Física, Universidade Federal do Rio de Janeiro, Caixa Postal 68528, Rio de Janeiro, 21945-970 Rio de Janeiro, Brazil
}

(Received 4 November 1999; published 23 June 2000)

\begin{abstract}
We study a field theory formulation of a fluid mechanical model. We implement the Hamiltonian formalism by using the BFFT conjecture in order to build a gauge invariant fluid field theory. We also generalize previous known classical dynamical field solutions for the fluid model.

PACS number(s): 11.10.Ef, 11.10.Lm, 11.30.Cp
\end{abstract}

\section{INTRODUCTION}

The basis of the canonical quantization for systems with infinite degrees of freedom has been the powerful character and applicability of the Dirac method [1]. Despite its current use in different systems, alternative formalisms have been developed in order to solve particular difficulties which come from Dirac's formulation [2]. One of these problems is the role of first- and second-class constraints when we identify the classical brackets as commutators. While first-class constraints are related to symmetries the second-class ones may imply some ambiguities when treated as quantum operators. The physical status of a theory is chosen by imposing complementary conditions which are given by the firstclass constraints. In order to avoid the presence of secondclass constraints we can separate it into first-class ones and gauge fixing terms; however, there is a special situation where the constraints are nonlinear so that this procedure fails [3].

An alternative way to circumvent this difficulty is to employ an interesting machinery proposed by Batalin, Fradkin, Fradkina, and Tyutin (BFFT) [4], which converts the secondclass constraints into first order ones by using auxiliary fields. Its applicability has been demonstrated in many different systems involving linear constraints $[5,6]$ and also in nonlinear cases $[3,7,8]$. As we expect, the implementation of the above mentioned method through the introduction of new fields gives rise to a kind of Wess-Zumino terms which turns the resulting effective theory gauge invariant.

In this paper we discuss the Hamiltonian formalism for a scalar field fluid theory from a BFFT method point of view. The fluid field theory has been introduced as a laboratory to study some classical aspects of membrane problem [9] but there are also other classical and quantum systems which can be described by this model [10-12]. We can mention, for instance, the hydrodynamical formulation of quantum mechanics [13] or a dimensional reduction of a relativistic scalar field theory [14].

Recently, Bazeia and Jackiw [10] have discussed this

\footnotetext{
*Email address: cesar@if.uff.br

†Email address: boschi@if.ufrj.br
}

model by making a careful analysis of the Galileo and Poincaré symmetries. In particular, they obtained dynamical solutions for the original fields by choosing a singular potential (see also Refs. [11,12]). In our study we use the BFFT method to build a gauge invariant theory to obtain the respective generators of the extended gauge transformations. As a consequence of this symmetry we show that for linear constraints, the Lagrangian is invariant in a similar way in respect to that discussed by Amorim and Barcelos [6] for chiral bosons theories.

We have organized this paper as follows. In Sec. II we present the fluid field theory as described by Bazeia and Jackiw. We show that their dynamical solutions for the singular potential can be generalized to other potentials leading to diverse physical systems. Section III is dedicated to the explanation of the BFFT method applied to the fluid field theory. Finally in Sec. IV we present an analysis of the results obtained and give our conclusions. We have also included two appendices where some technical calculations are given.

\section{THE MODEL: ITS SYMMETRIES AND SOLUTIONS}

Let us consider a fluid dynamical model [15] described by the following Lagrangian in $d$ dimensional $\mathbf{r}$ space, evolving in time $t$ :

$$
L=\int d^{d} r\left(\theta \dot{\rho}-\frac{1}{2} \rho \nabla \theta \cdot \nabla \theta-V(\rho)\right),
$$

where $\rho=\rho(t, \mathbf{r}), \theta=\theta(t, \mathbf{r})$ and the overdot means time differentiation. In a usual fluid mechanical model $\rho$ is the mass density and $\theta$ is the velocity potential, $\mathbf{v}=\nabla \theta$. Then, we have the equations of motion

$$
\begin{aligned}
& \dot{\rho}=\nabla(\rho \nabla \theta), \\
& \dot{\theta}=-\frac{1}{2}(\nabla \theta)^{2}-\frac{\delta}{\delta \rho} \int d^{d} r V .
\end{aligned}
$$

These equations of motion are recognized as the conventional ones for isentropic irrotational fluids [15]. As this system is a nonrelativistic one it has naturally the Galilean sym- 
metry [16], i.e., it is invariant under the Galilean group which generators are

$$
\begin{aligned}
H & =\int d^{d} r \mathcal{E}, \quad \mathcal{E}=\frac{1}{2} \rho(\nabla \theta)^{2}+V(\rho), \\
\mathbf{P} & =\int d^{d} r \mathcal{P}, \quad \mathcal{P}=\rho \nabla \theta, \\
J^{i j} & =\int d^{d} r \mathcal{J}^{i j}, \quad \mathcal{J}^{i j}=r^{i} \mathcal{P}^{j}-r^{j} \mathcal{P}^{i}, \\
\mathbf{B} & =\int d^{d} r \mathcal{B}, \quad \mathcal{B}=t \mathcal{P}-\mathbf{r} \rho, \\
N & =\int d^{d} r \rho,
\end{aligned}
$$

which, respectively, give time and space translation and space rotation, in addition to the Galileo boost and "charge" generator $N$ which in this case is the total mass of the fluid, being naturally conserved. The Poisson brackets of these generators close under an algebra corresponding to the Galileo group. For instance,

$$
\left\{B^{i}, P^{j}\right\}=\delta^{i j} N .
$$

A connection with the membrane problem [9] is done when $d=2$ and

$$
V(\rho)=\frac{g}{\rho},
$$

where $g$ is the coupling constant (this potential also connects the fluid field theory problem to $d$ branes in $d+1$ space dimensions [11,12]). In this case, the action $I=\int d t L$ is invariant under time rescaling $t \rightarrow e^{w} t$ generated by (dilation)

$$
D=\int d^{d} r(t \mathcal{E}-\rho \theta) .
$$
[10]

Another symmetry of this action is given implicitly by

$$
\begin{aligned}
& t \rightarrow T(t, \mathbf{r})=t+\mathbf{w} \cdot \mathbf{r}+\frac{\mathbf{1}}{\mathbf{2}} \mathbf{w}^{2} \theta(\mathbf{T}, \mathbf{R}), \\
& \mathbf{r} \rightarrow \mathbf{R}(t, \mathbf{r})=\mathbf{r}+\mathbf{w} \theta(T, R),
\end{aligned}
$$

where

$$
\theta(T, R)=\theta(t, \mathbf{r}-\mathbf{w} t)+\mathbf{w} \cdot \mathbf{r}-\frac{1}{2} \mathbf{w}^{2} t
$$

which are generated by

$$
\mathbf{G}=\int d^{d} r(\mathbf{r} \mathcal{E}-\theta \mathcal{P}) .
$$

The geometrical meaning of $\mathbf{G}$ is not clear, as pointed out in Ref. [10]; however, one should note that $D$ and $\mathbf{G}$ depend on the velocity potential $\theta$ which is meaningful only in the irrotational case. They speculated that, since the fluid field model corresponds to a gauge-fixed version of the relativistic membrane in the lightcone, the symmetry generated by $\mathbf{G}$ may be a residual gauge invariance of that model. As is well known, the Galileo group (2.4)-(2.8) together with the generators (2.11) and (2.12) defined in $(d+1)$ dimensions is isomorphic to a Poincaré group defined in $(d+1,1)$ dimensions [17].

Bazeia and Jackiw presented some solutions for this system with the potential (2.10) which are

$$
\begin{aligned}
& \theta(t, \mathbf{r})=-\frac{r^{2}}{2(d-1) t}, \\
& \rho(t, \mathbf{r})=\sqrt{\frac{2 g}{d}}(d-1) \frac{|t|}{r}
\end{aligned}
$$

valid for $d>1$. They discuss other solutions for the free case (a particular case of the above solution) and also some solutions for $d=1$, which are not our main concern here. Other solutions in different dimensions can also be found in Ref. [11].

Here we note that it is possible to extend the above results considering now the following potential:

$$
V(\rho)=\frac{g}{\rho^{n}}
$$

which describe ideal polytropic gases, i.e., a gas in which the pressure is proportional to a power of the density [18], for which we find the solutions

$$
\begin{aligned}
& \theta(t, \mathbf{r})=-\frac{r^{2}}{[d(n+1)-2] t}, \\
& \rho(t, \mathbf{r})=\left\{\frac{n g[d(n+1)-2]^{2} t^{2}}{d(n+1) r^{2}}\right\}^{1 /(n+1)},
\end{aligned}
$$

which are valid in $d>1$ space dimensions, generalizing the solutions obtained by Bazeia and Jackiw [10]. Their solution is a particular case of the above class of solutions which can be recovered when we take $n=1$ in the above equations. As we are going to show in the following section, the above fluid field theory also admits a gauge symmetry which is respected by a general potential $V(\rho)$.

\section{BFFT QUANTIZATION AND GAUGE SYMMETRY}

Let us now construct a gauge invariant version of the model described above using the method developed by Batalin, Fradkin, Fradkina, and Tuyutin (BFFT) [4] which transforms second-class constraints into first-class ones [6].

Considering the Lagrangian (2.1) we obtain the primary constraints

$$
\begin{aligned}
& \chi_{\rho}=\Pi_{\rho}-\theta \approx 0, \\
& \chi_{\theta}=\Pi_{\theta} \approx 0
\end{aligned}
$$


which satisfy the algebra

$$
\left\{\chi_{\rho}, \chi_{\theta}\right\}=-\epsilon_{\rho \theta} \delta(x-y) \equiv \Delta_{\rho \theta}(x, y) .
$$

Then, the primary Hamiltonian is given by

$$
\begin{aligned}
H_{p}= & \int d^{d} r\left(\Pi_{\rho} \dot{\rho}+\Pi_{\theta} \dot{\theta}-L+\lambda_{\rho} \chi_{\rho}+\lambda_{\theta} \chi_{\theta}\right) \\
= & \int d^{d} r\left(\left(\Pi_{\rho}-\theta\right) \dot{\rho}+\Pi_{\theta} \dot{\theta}+\frac{1}{2} \rho(\nabla \theta)^{2}+V(\rho)\right. \\
& \left.+\lambda_{\rho} \chi_{\rho}+\lambda_{\theta} \chi_{\theta}\right) \\
= & \int d^{d} r\left(\frac{1}{2} \rho(\nabla \theta)^{2}+V(\rho)+\tilde{\lambda}_{\rho} \chi_{\rho}+\tilde{\lambda}_{\theta} \chi_{\theta}\right),
\end{aligned}
$$

where we defined $\tilde{\lambda_{\rho}}=\lambda_{\rho}+\dot{\rho}, \tilde{\lambda_{\theta}}=\lambda_{\theta}+\dot{\theta}$. The consistency condition for the constraints determine the fields $\tilde{\lambda}_{\rho}, \widetilde{\lambda}_{\theta}$ and there are no other constraints.

Before we implement the BFFT method it is necessary here to make a brief review of it. For a more comprehensive and elegant discussion see Refs. [4,6]. Let us now begin by extending the phase space including the new fields $\varphi_{\rho}$ and $\varphi_{\theta}$ which satisfy the algebra

$$
\left\{\varphi_{\rho}, \varphi_{\theta}\right\}=\omega_{\rho \theta}(x, y)
$$

such that the new constraints $\Omega_{\rho}, \Omega_{\theta}$ should be first class and could be written in general as

$$
\Omega_{\beta}=\chi_{\beta}+\sigma_{\beta \alpha} \varphi^{\alpha},
$$

where $\sigma_{\beta \alpha}=\sigma_{\beta \alpha}(\rho, \theta)$. The central idea of the BFFT method is to write the first-class constraints in terms of the second-class ones as

$$
\Omega_{\beta}=\sum_{n=0}^{\infty} \chi_{\beta}^{(n)},
$$

with the condition $\chi_{\beta}^{(0)} \equiv \chi_{\beta}$. So, $\chi_{\beta}^{(n)}$ is of nth order in the field $\varphi_{\alpha}$. The new constraints defined by Eqs. (3.6), (3.7) satisfy the relation $\left\{\Omega_{\alpha}, \Omega_{\beta}\right\}=0$ and then

$$
\begin{gathered}
\left\{\chi_{\alpha}, \chi_{\beta}\right\}_{(\rho, \theta)}+\left\{\chi_{\alpha}^{(1)}, \chi_{\beta}^{(1)}\right\}_{(\varphi)}=0 \\
\left\{\chi_{\alpha}, \chi_{\beta}^{(1)}\right\}_{(\rho, \theta)}+\left\{\chi_{\alpha}^{(1)}, \chi_{\beta}\right\}_{(\rho, \theta)}+\left\{\chi_{\alpha}^{(1)}, \chi_{\beta}^{(2)}\right\}_{(\varphi)} \\
+\left\{\chi_{\alpha}^{(2)}, \chi_{\beta}^{(1)}\right\}_{(\varphi)}=0 \\
\left\{\chi_{\alpha}, \chi_{\beta}^{(2)}\right\}_{(\rho, \theta)}+\left\{\chi_{\alpha}^{(1)}, \chi_{\beta}^{(1)}\right\}_{(\rho, \theta)}+\left\{\chi_{\alpha}^{(2)}, \chi_{\beta}\right\}_{(\varphi)} \\
+\left\{\chi_{\alpha}^{(1)}, \chi_{\beta}^{(3)}\right\}_{(\varphi)}+\left\{\chi_{\alpha}^{(2)}, \chi_{\beta}^{(2)}\right\}_{(\varphi)}+\left\{\chi_{\alpha}^{(3)}, \chi_{\beta}^{(1)}\right\}_{(\varphi)}=0
\end{gathered}
$$

Here, we are using the notation $\{,\}_{(\rho, \theta)},\{,\}_{(\varphi)}$ referring to the Poisson brackets of the pairs $(\rho, \theta)$ and $\left(\varphi_{\rho}, \varphi_{\theta}\right)$. From Eqs. (3.3), (3.5), (3.6), (3.8) and using that $\left\{\Omega_{\alpha}, \Omega_{\beta}\right\}=0$ we have

$$
\Delta_{\rho \theta}=-\sigma_{\rho \alpha} \omega^{\alpha \beta} \sigma_{\theta \beta},
$$

which for the fluid field problem can be written as

$$
\begin{aligned}
\Delta_{\rho \theta} & =-\epsilon_{\rho \theta} \delta(x-y) \\
& =-\int d z d z^{\prime} \sigma_{\rho \alpha}(x, z) \omega^{\alpha \beta}\left(z, z^{\prime}\right) \sigma_{\theta \beta}\left(y, z^{\prime}\right) .
\end{aligned}
$$

As $\omega^{\alpha \beta}$ is obtained from second-class constraints $\varphi_{\alpha}$ we can choose $\omega^{\alpha \beta}=\epsilon_{\alpha \beta} \delta(x-y)$ which implies $\sigma_{\rho \alpha}=\epsilon_{\rho \alpha} \delta(z-x)$ so that

$$
\begin{aligned}
\Omega_{\rho}(x) & =\chi_{\rho}(x)+\int d z \delta(z-x) \varphi_{\theta}(z) \\
& =\chi_{\rho}(x)+\sigma_{\rho \theta} \varphi^{\theta}(x)
\end{aligned}
$$

and similarly

$$
\Omega_{\theta}(x)=\chi_{\theta}(x)+\sigma_{\theta \rho} \varphi^{\rho}(x) .
$$

The next step is to include corrections to the canonical Hamiltonian. We remark that in this formalism any dynamical function $A(\rho, \theta)$ can also be properly modified in order to be strong involutive with first-order constraints. So, if $\widetilde{A}(\rho, \theta, \varphi)$ is this quantity we have

$$
\left\{\Omega_{\rho}, A\right\}=0
$$

with the boundary condition

$$
\widetilde{A}(\rho, \theta, 0)=A(\rho, \theta) .
$$

In order to generate $\widetilde{A}$ we can repeat the same steps for the obtainment of $\Omega_{\rho}$ above, i.e., we consider the expansion

$$
\widetilde{A}=\sum_{n=0}^{\infty} A^{(n)},
$$

where $A^{(n)}$ is a term of order $n$ in the field $\varphi$. Consequently,from Eqs. (3.8)-(3.10) rewritten for $A^{(n)}$ and the condition $A^{(0)}=A$, we have

$$
A^{(1)}=-\varphi^{\alpha} \omega_{\alpha \beta} \sigma^{\beta \gamma}\left\{\chi_{\gamma}, A\right\},
$$

where $\omega_{\alpha \beta}=\left(\omega^{\alpha \beta}\right)^{-1}$ and $\sigma^{\beta \gamma}=\left(\sigma_{\beta \gamma}\right)^{-1}$. An equation analogous to Eq. (3.9) for $A^{(2)}$ gives

$$
\left\{\chi_{\rho}^{(1)}, A^{(2)}\right\}=-G_{\rho}^{(1)}
$$

such that

$$
G_{\rho}^{(1)}=\left\{\chi_{\rho}, A^{(1)}\right\}_{(\rho, \theta)}+\left\{\chi_{\rho}^{(1)}, A\right\}_{(\rho, \theta)}+\left\{\chi_{\rho}^{(2)}, A^{(1)}\right\}_{(\varphi)} .
$$

Then, we have, for $A^{(2)}$,

$$
A^{(2)}=-\frac{1}{2} \varphi^{\alpha} \omega_{\alpha \beta} \sigma^{\beta \gamma} G_{\gamma}^{(1)}
$$


and in general, for $n \geqslant 1$,

$$
A^{(n+1)}=-\frac{1}{n+1} \varphi^{\alpha} \omega_{\alpha \beta} \sigma^{\beta \gamma} G_{\gamma}^{(n)}
$$

with the auxiliary condition $G_{\rho}^{(0)}=\left\{\chi_{\rho}, A\right\}$ so that

$$
\begin{aligned}
G_{\rho}^{(n)}= & \sum_{m=0}^{n}\left\{\chi_{\rho}^{(n+m)}, A^{(m)}\right\}_{(\rho, \theta)}+\sum_{m=0}^{n-2}\left\{\chi_{\rho}^{(n-m)}, A^{(m+2)}\right\}_{(\varphi)} \\
& +\left\{\chi_{\rho}^{(n+1)}, A^{(1)}\right\}_{(\varphi)} .
\end{aligned}
$$

\section{A. Particular case: $\sigma_{\alpha \beta}$ independent of $(\rho, \theta)$}

In the particular case where $\sigma_{\alpha \beta}$ does not depend on $(\rho, \theta)$ the $A^{(n)}$ are still given by Eq. (3.22) but

$$
G_{\rho}^{(n)}=\left\{\chi_{\rho}, A^{(n)}\right\}
$$

Let us analyze this particular case further with the additional hypothesis that the second-class constraints are all linear. Then, we can write

$$
\begin{aligned}
A^{(n+1)} & =-\frac{1}{n+1} \varphi^{\alpha} \omega_{\alpha \beta} \sigma^{\beta \gamma}\left\{\chi_{\gamma}, A^{(n)}\right\} \\
& =-\frac{1}{n+1} \varphi^{\alpha} \omega_{\alpha \beta} \sigma^{\beta \gamma}\left\{\chi_{\gamma}, z^{i}\right\} \frac{\partial}{\partial z^{i}} A^{(n)} \\
& =-\frac{1}{n+1} \varphi^{\alpha} k_{\alpha}^{i} \partial_{i} A^{(n)}
\end{aligned}
$$

where we have used the Jacobi identity and the defined $z^{i}$ is a generalized phase space coordinate, in the sense that it could be either a canonical coordinate or a canonical momentum. Note that $k_{\alpha}^{i} \equiv \omega_{\alpha \beta} \sigma^{\beta \gamma}\left\{\chi_{\gamma}, z^{i}\right\}$ is a constant matrix since the constraints are linear, $\left\{\chi_{\gamma}, z^{i}\right\}=$ const and $\sigma^{\beta \gamma}$ is a constant matrix too in this particular case. Using Eq. (3.25) iteratively one finds

$$
A^{(n)}=\frac{(-1)^{n}}{n !}\left(\varphi^{\alpha} k_{\alpha}^{i} \partial_{i}\right)^{n} A
$$

so that

$$
\begin{aligned}
\widetilde{A}(\rho, \theta ; \varphi) & \equiv \sum_{n=0}^{\infty} A^{(n)} \\
& =\sum_{n=0}^{\infty} \frac{(-1)^{n}}{n !}\left(\varphi^{\alpha} k_{\alpha}^{i} \partial_{i}\right)^{n} A=\exp \left(\varphi^{\alpha} k_{\alpha}^{i} \partial_{i}\right) A
\end{aligned}
$$

and then in this case the operator $\widetilde{A}$ will be of the form

$$
\widetilde{A}\left(z^{i}, \varphi^{\alpha}\right)=A\left(z^{i}-\varphi^{\alpha} k_{\alpha}^{i}\right) .
$$

\section{B. General case: $\sigma_{\alpha \beta}$ as as function of $(\rho, \theta)$}

Let us now return to the discussion of the general case and construct the extended Hamiltonian. Noting that for $n$ $\geqslant 2, \chi_{\rho}^{(n)}=0$, so that

$$
H_{c}^{(n+1)}=-\frac{1}{n+1} \int d x d y d z \varphi_{\alpha}(x)\left(\omega_{\alpha \beta}\right)^{-1}\left(\sigma_{\beta \gamma}\right)^{-1} G_{\gamma}^{(n)}
$$

where $G_{\gamma}^{(n)}$ is given by

$$
G_{\gamma}^{(n)}=\left\{\chi_{\gamma}, H_{c}^{(n)}\right\}
$$

Since $\left(\omega_{\alpha \beta}\right)^{-1}$ and $\left(\sigma_{\beta \gamma}\right)^{-1}$ are proportional to Dirac delta functions, we have

$$
H_{c}^{(0)}=\int d x\left[\frac{1}{2} \rho(\nabla \theta)^{2}-V(\rho)\right]
$$

so that

$$
G_{\rho}^{(0)}=\left\{\chi_{\rho}, H_{c}^{(0)}\right\}=-\frac{1}{2}(\nabla \theta)^{2}+\partial_{\rho} V
$$

and also

$$
G_{\theta}^{(0)}=\left\{\chi_{\theta}, H_{c}^{(0)}\right\}=-\frac{1}{2} \rho \nabla^{2} \theta
$$

so that the correction $H_{c}^{(1)}$ is given by

$$
H_{c}^{(1)}=-\int d x\left\{\left[\frac{1}{2}(\nabla \theta)^{2}-\partial_{\rho} V\right] \varphi_{\rho}-\frac{1}{2}\left(\rho \nabla^{2} \theta\right) \varphi_{\theta}\right\} \text {. }
$$

Continuing the iteration process and summing up all the contributions we find that the canonical Hamiltonian is then given by (see the Appendix A)

$$
\begin{aligned}
H_{c}= & H_{c}^{(0)}+\int d x\left[-\lambda_{\theta} \varphi_{\rho}+\lambda_{\rho} \varphi_{\theta}-\frac{1}{2}\left(\partial_{\rho}^{2} V\right) \varphi_{\rho}^{2}+\cdots\right. \\
& \left.+\frac{(-1)^{n}}{n !}\left(\partial_{\rho}^{n} V\right) \varphi_{\rho}^{n}+\cdots\right] \\
= & H_{c}^{(0)}+\int d x\left[-\lambda_{\theta} \varphi_{\rho}+\lambda_{\rho} \varphi_{\theta}+\sum_{n=0}^{\infty} \frac{(-1)^{n+2}}{(n+2) !}\right. \\
& \left.\times\left(\partial_{\rho}^{(n+2)} V\right) \varphi_{\rho}^{(n+2)}\right],
\end{aligned}
$$

where the term corresponding to $H_{c}^{(1)}$ is contained in $\lambda_{\theta}$ $=\frac{1}{2}(\nabla \theta)^{2}-\partial_{\rho} V$. Note that from the definition (3.6) the firstclass constraints are given by

$$
\begin{aligned}
& \Omega_{\rho}=\chi_{\rho}+\sigma_{\rho \theta} \varphi^{\theta}, \\
& \Omega_{\theta}=\chi_{\theta}+\sigma_{\theta \rho} \varphi^{\rho},
\end{aligned}
$$


where $\sigma_{\rho \theta}=-\sigma_{\theta \rho}=1$. Let us now go back to the canonical Hamiltonian Eq. (3.35) and analyze its last term. We note that

$$
\begin{aligned}
\sum_{n=0}^{\infty} & \frac{(-1)^{n+2}}{(n+2) !}\left(\partial_{\rho}^{(n+2)} V\right) \varphi_{\rho}^{(n+2)} \\
& =\sum_{n=0}^{\infty} \Theta(n-1) \frac{(-1)^{n}}{n !}\left(\partial_{\rho}^{n} V\right) \varphi_{\rho}^{n} \\
& =-V(\rho)+\varphi_{\rho} \partial_{\rho} V(\rho)-e^{-\varphi_{\rho} \partial} V(\rho),
\end{aligned}
$$

where $\Theta(x)$ is the Heavside function. This way we have

$$
\begin{aligned}
H_{c}= & \int d x\left[-\lambda_{\theta} \varphi_{\rho}+\lambda_{\rho} \varphi_{\theta}-e^{-\varphi_{\rho} \partial_{\rho}} V(\rho)\right] \\
= & \int d x\left[\frac{1}{2} \rho(\nabla \theta)^{2}-\frac{1}{2}(\nabla \theta)^{2} \varphi_{\rho}-\frac{1}{2} \rho \nabla^{2} \theta \varphi_{\theta}\right. \\
& \left.-e^{-\varphi_{\rho} \partial_{\rho}} V(\rho)\right]
\end{aligned}
$$

In order to find the corresponding Lagrangian, we identify $\varphi \equiv \varphi_{\rho}$ and $\Pi_{\varphi} \equiv \varphi_{\theta}$ as a pair of canonical conjugate coordinates and write the generating functional

$$
\begin{aligned}
\mathcal{Z}= & \mathcal{N} \int[d \rho]\left[d \Pi_{\rho}\right][d \theta]\left[d \Pi_{\theta}\right][d \varphi]\left[d \Pi_{\varphi}\right] \\
& \times \delta\left(\Pi_{\rho}-\theta+\Pi_{\varphi}\right) \delta\left(\Pi_{\theta}-\varphi\right) \\
& \times \exp \left\{i \int d x \left[\Pi_{\rho} \dot{\rho}+\Pi_{\theta} \dot{\theta}+\Pi_{\varphi} \dot{\varphi}-\frac{1}{2} \rho(\nabla \theta)^{2}\right.\right. \\
& \left.\left.+\frac{1}{2}(\nabla \theta)^{2} \varphi+\frac{1}{2} \rho \nabla^{2} \theta \Pi_{\varphi}+e^{-\varphi \partial_{\rho}} V(\rho)\right]\right\},
\end{aligned}
$$

where the delta functions represent the first-class constraints. Noting that

(i)

$$
\begin{aligned}
& \int\left[d \Pi_{\theta}\right] \delta\left(\Pi_{\theta}-\varphi\right) \exp \left\{i \int d x \Pi_{\theta} \dot{\theta}\right\} \\
& =\exp \left\{i \int d x \varphi \dot{\theta}\right\}, \\
& \int\left[d \Pi_{\rho}\right] \delta\left(\Pi_{\rho}-\theta-\Pi_{\varphi}\right) \exp \left\{i \int d x \Pi_{\rho} \dot{\rho}\right\} \\
& =\exp \left\{i \int d x\left(\Pi_{\varphi}+\theta\right) \dot{\rho}\right\}
\end{aligned}
$$

(ii)

and substituting these results into the generating functional we find

$$
\begin{aligned}
\mathcal{Z}= & \mathcal{N} \int[d \rho][d \theta][d \varphi]\left[d \Pi_{\varphi}\right] \exp \left\{i \int d x \left[\theta \dot{\rho}+\Pi_{\theta} \dot{\theta}+\Pi_{\varphi} \dot{\varphi}\right.\right. \\
& \left.\left.-\frac{1}{2} \rho(\nabla \theta)^{2}+\frac{1}{2}(\nabla \theta)^{2} \varphi+\frac{1}{2} \rho \nabla^{2} \theta \Pi_{\varphi}+e^{-\varphi \partial_{\rho} V(\rho)}\right]\right\} .
\end{aligned}
$$

The functional integral over $\Pi_{\varphi}$ gives

$$
\begin{aligned}
\int & {\left[d \Pi_{\varphi}\right] \exp \left\{i \int d x \Pi_{\varphi}\left(\dot{\rho}+\dot{\varphi}+\frac{1}{2} \rho \nabla^{2} \theta\right)\right\} } \\
= & \delta\left(\dot{\rho}+\dot{\varphi}+\lambda_{\rho}\right)
\end{aligned}
$$

so that the generating functional reads

$$
\begin{aligned}
\mathcal{Z}= & \mathcal{N} \int[d \rho][d \theta][d \varphi] \delta\left(\dot{\rho}+\dot{\varphi}+\lambda_{\rho}\right) \\
& \times \exp \left\{i \int d x \left[\theta \dot{\rho}+\varphi \dot{\theta}-\frac{1}{2}(\rho-\varphi)(\nabla \theta)^{2}\right.\right. \\
& \left.\left.+e^{-\varphi \partial_{\rho}} V(\rho)\right]\right\}
\end{aligned}
$$

and then the extended Lagrangian density is given by

$$
\widetilde{\mathcal{L}}=\theta \dot{\rho}+\varphi \dot{\theta}-\frac{1}{2}(\rho-\varphi)(\nabla \theta)^{2}+e^{-\varphi \partial_{\rho}} V(\rho) .
$$

In the limit in which the auxiliary field vanishes, $\varphi \rightarrow 0$, we get back the original Lagrangian (2.1) as it should. Let us now look at the last term of this Lagrangian which involves the potential $V(\rho)$. Assuming that the potential function can be expanded in a power series we have

$$
e^{-\varphi \partial_{\rho} V(\rho)=V(\rho-\varphi)}
$$

So, we can apply the above equation for a wide range of functions $V(\rho)$, as for example, the potentials discussed in Sec. II.

Then, the extended Lagrangian density after a partial integration becomes

$$
\widetilde{\mathcal{L}}=-(\rho-\varphi) \dot{\theta}-\frac{1}{2}(\rho-\varphi)(\nabla \theta)^{2}+V(\rho-\varphi)
$$

so that the Lagrangian is invariant under the exchange $\rho$ $\rightarrow \rho-\varphi$ which is the gauge symmetry of the model. Since the first-class $\Omega$ is strongly involutive with canonical Hamiltonian (see the Appendix B) it is easy to check the invariance of $\widetilde{\mathcal{L}}(\rho, \theta, \varphi)$.

Now, we can look at the consequences of this gauge symmetry on the previous known symmetries for the fluid dynamical model. The Galileo and Poincare groups in the gauged model can be obtained from the the density generators of the nongauge model, Eqs. (2.4)-(2.8), (2.11), and (2.12) simply through the shift $\widetilde{\mathcal{O}}=e^{-\varphi \partial_{\rho} \mathcal{O}}$, so that the original Galileo and Poincare invariances of the fluid field model 
are preserved by the introduction of the auxiliary field which bring to it a gauge symmetry.

\section{CONCLUSIONS}

In this article we have studied the fluid field theory [9] for which we found a class of classical solutions which recover previous known particular cases [10].

Then, by means of the BFFT formalism [4] we extended the original phase space by including new fields which permitted the transformation of the set of second-class constraints into a first-class one. We have analyzed a situation where we found an extended gauge symmetry for an arbitrary potential with linear constraints and a kind of a WessZumino Lagrangian was built. As a result we have obtained a new gauge invariant system. This new system may be of interest to the membrane problem related to Lagrangian (2.1) since that formulation corresponds to a gauge fixed version in the light-cone gauge. As a final remark it is important to mention that the procedure discussed here could also be applied successfully to a situation where nonlinear constraints were involved, as is well known in general for the BFFT method.

\section{ACKNOWLEDGMENTS}

H.B.-F. would like to acknowledge interesting discussions with D. Bazeia, R. Jackiw, J. Hoppe, and C. Sigaud on the model presented in section II and CTP-MIT where part of this work was done. C.P.N. was partially supported by $\mathrm{CNPq}$ and H.B.-F. by CNPq, FINEP, and FUJB (Brazilian research agencies).

\section{APPENDIX A}

In this Appendix we give some details of the iteration process necessary to construct the canonical Hamiltonian in the case of the linear constraint discussed in Sec. III. Using Eqs. (3.29) and (3.30) we found the first correction, $H_{c}^{(1)}$. For the next correction we have

$$
G_{\rho}^{(1)}=\left\{\chi_{\rho}, H_{c}^{(1)}\right\}=-\frac{\delta}{\delta \rho} H_{c}^{(1)}=-\left(\partial_{\rho}^{2} V\right) \varphi_{\rho}-\frac{1}{2}\left(\nabla^{2} \theta\right) \varphi_{\theta}
$$

while

$$
G_{\theta}^{(1)}=\left\{\chi_{\theta}, H_{c}^{(1)}\right\}=-\frac{\delta}{\delta \theta} H_{c}^{(1)}=+\frac{1}{2}\left(\nabla^{2} \theta\right) \varphi_{\rho}
$$

so that

$$
H_{c}^{(2)}=-\frac{1}{2} \int d x\left(\partial_{\rho}^{2} V\right) \varphi_{\rho}^{2}
$$

For third correction to the canonical Hamiltonian, we find

$$
G_{\rho}^{(2)}=\left\{\chi_{\rho}, H_{c}^{(2)}\right\}=-\frac{\delta}{\delta \rho} H_{c}^{(2)}=\frac{1}{2}\left(\partial_{\rho}^{3} V\right) \varphi_{\rho}^{2}
$$

and

$$
G_{\theta}^{(2)}=-\frac{\delta}{\delta \theta} H_{c}^{(1)}=0
$$

so that

$$
H_{c}^{(3)}=\frac{1}{2.3} \int d x\left(\partial_{\rho}^{3} V\right) \varphi_{\rho}^{3}
$$

For the next term we have

$$
G_{\rho}^{(3)}=\left\{\chi_{\rho}, H_{c}^{(3)}\right\}=-\frac{\delta}{\delta \rho} H_{c}^{(3)}=\frac{1}{2.3}\left(\partial_{\rho}^{4} V\right) \varphi_{\rho}^{3}
$$

and $G_{\theta}^{(n)}=0$ for $n \geqslant 2$ so that

$$
H_{c}^{(4)}=-\frac{1}{2.3 .4} \int d x\left(\partial_{\rho}^{4} V\right) \varphi_{\rho}^{4}
$$

\section{APPENDIX B}

Let us show here that the first-class constraints $\Omega_{\alpha}$ are strongly involutive in respect to the canonical Hamiltonian $H_{c}\left(\rho, \theta, \varphi_{\rho}, \varphi_{\theta}\right)$, i.e., $\left\{\Omega_{\alpha}, H_{c}\right\}=0$. First note that from definition of $\Omega_{\rho}, \Omega_{\theta}$, Eqs. (3.36), (3.37), and the canonical Hamiltonian Eq. (3.39), we have

$$
\begin{aligned}
\left\{\Omega_{\rho}, H_{c}\right\}= & \left\{\Omega_{\rho}, H_{c}^{(0)}\right\}+\left\{\lambda_{\rho}, \Omega_{\rho}\right\} \varphi_{\theta}-\lambda_{\theta}\left\{\Omega_{\rho}, \varphi_{\rho}\right\} \\
& -\left\{\lambda_{\theta}, \Omega_{\rho}\right\} \varphi_{\rho}-\left\{\Omega_{\rho}, e^{-\varphi_{\rho} \partial_{\rho} V}\right\}
\end{aligned}
$$

where we have used the fact that $\left\{\Omega_{\rho}, \varphi_{\theta}\right\}=0$. Then,

$$
\left\{\Omega_{\rho}, H_{c}\right\}=\left\{\lambda_{\rho}, \Omega_{\rho}\right\} \varphi_{\theta}-\left\{\lambda_{\theta}, \Omega_{\rho}\right\} \varphi_{\rho} .
$$

Now, using $\varphi_{\theta}=\Omega_{\rho}-\chi_{\rho}$ and $\varphi_{\rho}=\Omega_{\theta}-\chi_{\theta}$ we have

$$
\begin{aligned}
\left\{\Omega_{\rho}, H_{c}\right\}= & \left\{\lambda_{\rho}, \Omega_{\rho}\right\}\left(\Omega_{\rho}-\chi_{\rho}\right)-\left\{\lambda_{\theta}, \Omega_{\rho}\right\}\left(\Omega_{\theta}-\chi_{\theta}\right) \\
= & \left(\left\{\lambda_{\rho}, \Omega_{\rho}\right\}-\left\{\lambda_{\rho}, \chi_{\rho}\right\}\right) \Omega_{\rho}+\left(\left\{\lambda_{\theta}, \Omega_{\theta}\right\}\right. \\
& \left.-\left\{\lambda_{\theta}, \chi_{\theta}\right\}\right) \Omega_{\rho}=\left\{\lambda_{\rho}, \Omega_{\rho}\right\} \Omega_{\rho}+\left\{\lambda_{\theta}, \Omega_{\theta}\right\} \Omega_{\rho}
\end{aligned}
$$

and then we find

$$
\left\{\Omega_{\rho}, H_{c}\right\}=0 \text {. }
$$

An analogous result can be found for $\Omega_{\theta}$, proving our original statement.
[1] P. A. M. Dirac, Can. J. Phys. 2, 129 (1950); Lectures on Quantum Mechanics (Yeshiva University Press, New York, 1964).

[2] M. Henneaux and C. Teitelboim, Quantization of Gauge Systems (Princeton University Press, Princeton, 1992).
[3] See, for example, R. Banerjee and J. Barcelos-Neto, Nucl. Phys. B499, 453 (1997); J. Barcelos-Neto and W. Oliveira, Phys. Rev. D 56, 2257 (1997).

[4] I. A. Batalin and E. S. Fradkin, Phys. Lett. B 180, 157 (1986); 
Nucl. Phys. B279, 514 (1987); I. A. Batalin, E. S. Fradkin, and T. E. Fradkina, ibid. B314, 158 (1989); B323, 734 (1989); I. A. Batalin and I. V. Tyutin, Int. J. Mod. Phys. A 6, 3255 (1991).

[5] T. Fujikawa, Y. Igarashi, and J. Kubo, Nucl. Phys. B341, 695 (1990)

[6] R. Amorim and J. Barcelos-Neto, Phys. Lett. B 333, 413 (1994); Phys. Rev. D 53, 7129 (1996).

[7] N. Banerjee, R. Banerjee, and S. Gosh, Phys. Rev. D 49, 1996 (1994).

[8] Y-W. Kim, Y-J. Park, and K. D. Rothe, J. Phys. G 24, 953 (1998); Y-W. Kim and K. D. Rothe, Nucl. Phys. B511, 510 (1998).

[9] M. Bodermann and J. Hoppe, Phys. Lett. B 317, 315 (1993); 325, 359 (1994); J. Hoppe, ibid. 329, 10 (1994).
[10] D. Bazeia and R. Jackiw, Ann. Phys. (N.Y.) 270, 246 (1998).

[11] D. Bazeia, Phys. Rev. D 59, 085007 (1999).

[12] R. Jackiw and A. P. Polychronakos, "Faddeev Festschrift," Steklov Mathematical Institute Proceedings, hep-th/9809123; Commun. Math. Phys. 207, 107 (1999).

[13] E. Madelung, Z. Phys. 40, 322 (1926); C. Eckart, Phys. Rev. 54, 920 (1938); A. Schakel, Mod. Phys. Lett. B 10, 999 (1996).

[14] A. Jevicki, Phys. Rev. D 57, 5955 (1998).

[15] L. Landau and E. Lifchitz, Fluid Mechanics, 2nd ed. (Pergamon, Oxford, 1987).

[16] See, e.g., E. C. G. Sudarshan and N. Mukunda, Classical Dynamics: A Modern Perspective (Wiley, New York, 1974).

[17] L. Susskind, Phys. Rev. 165, 1535 (1968).

[18] See, e.g., S. Chandrasekhar, An Introduction to the Study of Stellar Structure (Dover, New York, 1967). 